\title{
Theoretically modelling photoionised regions with fractal geometry in three-dimension
}

\author{
Yifei Jin@, Lisa Kewley and Ralph Sutherland \\ Research School of Astronomy \& Astrophysics, The Australian National University, \\ Cotter Road, Weston Creek, 2611, ACT, Australia \\ email: Yifei. Jin@anu.edu.au
}

\begin{abstract}
Accurate predictions of the physics of interstellar medium (ISM) are vital for understanding galaxy formation and evolution. Modelling photoionized regions with complex geometry produces realistic ionization structures within the nebulae, providing the necessary physical predictions to interpret observational data. 3D photoionization codes built with Monte Carlo techniques provide powerful tools to produce the ionizing radiation field with fractal geometry. We present a high-resolution Monte Carlo modelling of a nebula with fractal geometry, and will further show how nebular geometry influences the emission-line behaviours. Our research has important implications for studies of emission-line ratios in high redshift galaxies.
\end{abstract}

Keywords. ISM: HII regions, galaxies: ISM, galaxies: starburst, galaxies: high-redshift.

\section{Nebular Geometry in Photoionisation Models}

The accurate interpretations of emission-line behaviours in galaxies largely depend on photoionisation models. Over the past three decades, the CLOUDY (Ferland et al. 2017) and the MAPPINGS codes (Sutherland et al. 2017) have been two widely-recognized self-consistent radiative transfer codes. The MAPPINGS code was also developed to resolve the physical structure and emission-line spectrum of both fast and slow shocks. However, both the CLOUDY code and the MAPPINGS code are built in plane parallel or spherical simplifications of nebular geometry, which limit their usage in modelling the realistic photoionised regions.

The Monte Carlo technique is a promising solution to 3D radiative transfer calculations. Several Monte Carlo radiative transfer codes have been developed and used in the nebular geometric studies, like MOCASSIN3D (Ercolano et al. 2005) and DIRTY (Gordon et al. 2001). So far, these nebular geometric studies focus on modelling the density bounded photoionised regions with simple density distributions, like bipolar or porous spherical density distributions. In this work, we model a photoionised region with complex threedimensional geometry, in order to investigate the geometric effect on the behaviour of nebular lines.

\section{Modelled Radiation Bounded Photoionised Region with Complex Geometry}

We adopt the MOCASSIN3D code to model a radiation bounded photoionised region in a three-dimensional ISM cube. The ISM has a lognormal density distribution in order to describe the turbulence in ISM. We place an O-star at the centre of the ISM cube. The ionising spectrum is selected from the CMFGEN stellar library. 

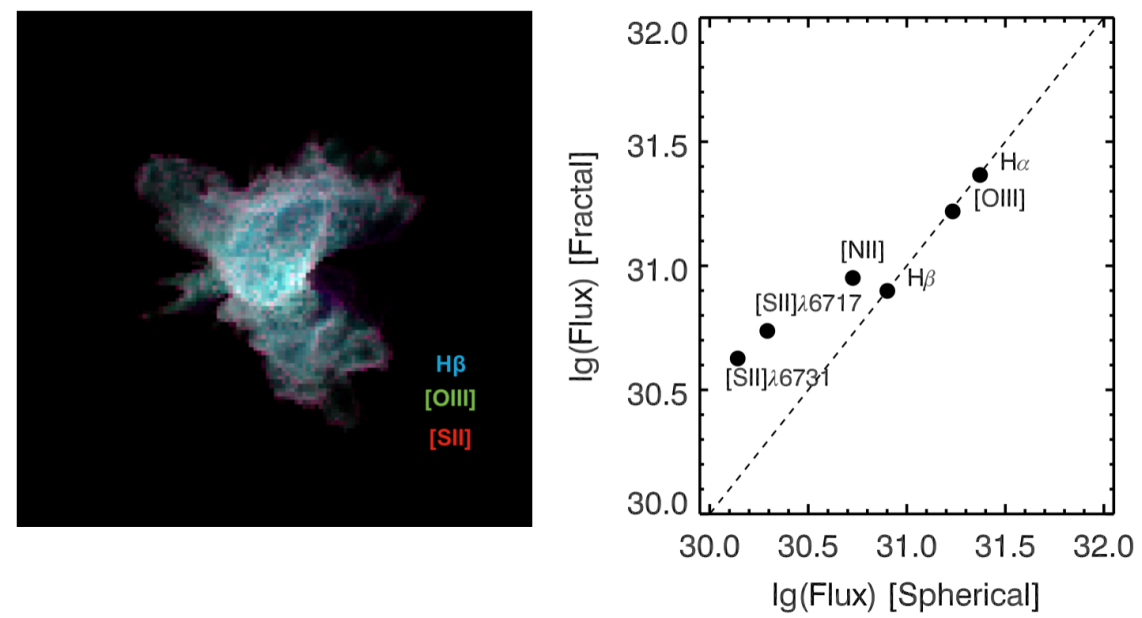

Figure 1. Left: The projected distributions of $\mathrm{H} \beta$, [O III] and [S II] emissions. Right: The comparison of integrated emission-line fluxes between the fractal model and the spherical simplified model.

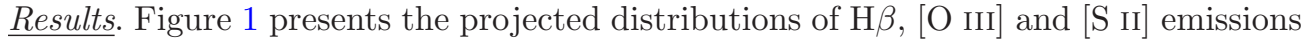
of our modelled photoionised region. The photoionised region has a twisted boundary, which is shaped by the fractal density distribution of ISM. Similar to the predication of $1 \mathrm{D}$ models, $\mathrm{H} \beta$ and [O III] emissions spread across the entire photoionised region, while $[\mathrm{S} \mathrm{II}]$ and $[\mathrm{N}$ II] preferentially locate at the boundary of the photoionised region. Compared with the equal-mass spherically simplified model, our geometric model shows higher [S II] and [N II] fluxes but similar $\mathrm{H} \alpha, \mathrm{H} \beta$ and [O III] fluxes. The increasing complexity of nebular geometry enhances the integrated flux of [S II] and [N II] but has little influence on $\mathrm{H} \beta$ and [O III] fluxes.

Implications and future. The 3D Monte Carlo photoionisation model with complex geometry provides a better predication of the emission-line behaviours in realistic galaxies, especially for high-redshift galaxies where the ISM is highly-turbulent. In order to fully understand the geometric effect on emission-line behaviours, systematic studies considering various nebular geometries are required in future.

\section{References}

Ercolano, B., Barlow, M. J., \& Storey, P. J. 2005, MNRAS, 362, 1038

Ferland, G. J., Chatzikos, M., Guzmán, F., Lykins, M. L., van Hoof, P. A. M., Williams, R. J. R., Abel, N. P., Badnell, N. R., Keenan, F. P., Porter, R. L., \& Stancil, P. C. 2017, Rev. Mexicana AyA, 53, 385

Gordon, K. D., Misselt, K. A., Witt, A. N., \& Clayton, G. C. 2001, ApJ, 551, 269

Sutherland, R. S. \& Dopita, M. A., et al. 2017, ApJS, 229, 34 